\title{
Análisis DOFA del sector logístico portuario de la ciudad de Cartagena ${ }^{1}$
}

\author{
Diógenes Alejandro Arrieta Rodríguez ${ }^{2}$ \\ Diego A. Cardona A. ${ }^{3}$
}

\section{Resumen}

En el presente artículo se realiza un análisis DOFA del sector logístico portuario de la ciudad de Cartagena de indias, la investigación se desarrolla en esta ciudad debido a la posición estratégica privilegia con la que cuenta la misma, lo que le permite el manejo de cargas hacia el sur de continente, de mismo modo que hacia el centro y norte América. La investigación de la cual deriva el artículo es de tipo descriptiva y analítica, y se basa en fuentes secundaria a través de las cuales se analiza el sector logístico portuario en la ciudad de Cartagena, y de igual forma se identifican los principales retos en materia competitiva del sector durante los próximos años. Como conclusión generalizada se pude decir que el puerto de Cartagena entra a desempeñar un papel importante, trascendental en el comportamiento económico de la región y del país en los próximos años, siempre y cuando se adopten las estrategias necesarias y pertinentes para poder cumplir con este compromiso y configurarse como Centro de Conexión Global por excelencia en el Caribe.

\section{Palabras Claves}

Sector logístico, Sector Portuario, Estructurada empresarial, DOFA

\begin{abstract}
In the present article a SWOT analysis of the port logistic sector of the city of Cartagena de Indias is carried out, the research is developed in this city due to the privileged strategic position it has, which allows the handling of loads towards the south of continent, in the same way as to the center and north America. The research from which the article derives is descriptive and analytical, and is based on secondary sources through which the port logistics sector in the city of Cartagena is analyzed, and in the same way, the main challenges in competitive matters are identified of the sector during the next years. As a general conclusion, we can say that the port of Cartagena has an important role to play, which is important in the economic behavior of the region and the country in the coming years, as long as the necessary and pertinent strategies are adopted to be able to fulfill this commitment. and be configured as the Global Connection Center par excellence in the Caribbean.
\end{abstract}

\section{Keywords}

Logistics sector, Port Sector, Structured business, DOFA.

\footnotetext{
1. Este artículo se deriva del trabajo de investigación titulado Análisis de la configuración estratégica de las empresas del sector logístico portuario de la ciudad de Cartagena, presentado con el objetivo de obtener el título de Magíster en Administración por la Universidad Tecnológica de Bolívar.

2. Magíster en Administración por la Universidad Tecnológica de Bolívar. Especialista en Finanzas Corporativas y Mercado de Capitales por la Universidad Pontificia Bolivariana. Administrador de Empresas por la Universidad de Cartagena. Docente de catedra de la Universidad de Cartagena E-mail: dioge_arrieta@hotmail.com

3. PhdC en Administración, Magister en Desarrollo Empresarial, Administrador de Empresas.
} 
Diógenes Alejandro Arrieta Rodríguez, Diego A. Cardona A.

\section{Introducción}

El sistema logístico portuario de Cartagena abarca a todas las instalaciones portuarias destinadas a la carga/descarga de todo tipo de mercancías, pasajeros, reparación y construcción de embarcaciones, turismo y otros servicios marítimos. También comprende los fondeadores y canales que permiten las maniobras de los buques y dan acceso a los atraques, así como los sistemas de comunicación y señalización para la seguridad de la navegación.

El sistema logístico portuario está conformado por modernas terminales para el manejo de carga y contenedores, la principal característica de la zona portuaria de Cartagena es su posición geográfica, ya que está ubicada en medio de uno de los centros industriales, turísticos y comerciales más importantes del país. Además de estar ubicado en una posición geográfica con características únicas tales como bahía, libre de la ruta de huracanes, en la mejor esquina de Colombia en la que se conecta desde el Caribe con el Atlántico y por Panamá con el Pacifico. Cuenta con dos canales de acceso, se comunica con el interior del país y con los puertos de Barranquilla y Santa Marta por medio de la carretera troncal del Caribe, y con el Río Magdalena a través del Canal del Dique (Bejarano, 2013). Adicionalmente, su cercanía con el canal de Panamá lo posicionan dentro del nodo de conectividad más importantes del mundo, por encima del Canal de Suez, Shanghái y Singapur, ya que le permite conectar a grandes centros de consumo europeos y norteamericanos (Martínez Ortiz et al., 2014).

La administración portuaria nacional está amparada por la Ley 01 de 1991, principalmente para solucionar los problemas de monopolio estatal, sobrecostos y el atraso en infraestructura, entre otros. Los puertos se entregaron en concesión, se autorizó la liquidación de Colpuertos y se crearon instituciones como las Sociedades Portuarias, la Superintendencia General de Puertos y empresas de operadores portuarios privados, que han llevado al sistema a buenos resultados y avances importantes en lo referente a tecnología y eficiencia.
En términos portuarios, Cartagena se constituyó como zona portuaria en 1533, sin embargo su primer muelle se construyó en 1935, a partir de esta fechas han sido muchos los cambios que se han realizado, catapultándolo como el principal puerto del Caribe colombiano, en el cual se moviliza una parte importante de la carga que ingresa al país. Cabe resaltar, que junto con los procesos de mejoramiento de la zona portuaria, la privatización ha estado implícita, administrada en su mayoría por la Sociedad Portuaria de Cartagena (Acosta, 2012).

En Colombia, en 2014, el sector portuario movió un total de 184,7 millones de toneladas, de este total el $43 \%$ correspondió al carbón, y en menor proporción al petróleo, con 30\%. Para este mismo año, la zona portuaria que más movió carga fue la de Ciénaga (41,8 millones de toneladas), seguido de la zona de Coveñas, Puerto Bolívar y en cuarto lugar la zona portuaria de Cartagena. Del total de la carga, las sociedades portuarias públicas movieron un 51\% ( 94,2 millones de toneladas), sobresaliendo la Sociedad Portuaria de Cartagena con 42,5 millones de toneladas y la Sociedad Portuaria de Buenaventura con 32 millones (ANDI, 2015). Es importante resaltar, el crecimiento que ha tenido el sector logístico portuario de Cartagena. En 2007, el número de empresas de este sector fue de 750 firmas, para 2011 fue de 850 empresas aproximadamente, $y$ para 2016 la cifra pasó a 14004. Es decir, que el número de empresas en este sector aumentaron en un $86,7 \%$, en estos últimos 10 años, lo que se traduce en un aumento promedio anualizado en más del $25 \%$ de los activos de este sector. En este sentido, es oportuno resaltar el aporte de este sector en la ciudad, y a la economía del país. Los últimos informes demuestran que en este sector se trasporta más del $50 \%$ de la carga que entra al país, del cual, el 70\% está destinado al trasbordo (Acosta, 2012).

En términos más específicos, el puerto de Cartagena está conformado por ocho muelles, cinco bodegas, cinco patios, entre otros. Esta infraestructura le permite atender barcos de 5.000 Teus 5. y capacidad para movilizar 1.200.000 Teus.

4. Cifra preliminar

5. Las siglas TEU (Por sus siglas en ingles hacen referencia al término Twenty-foot Equivalent Unit, que significa Unidad Equivalente a Veinte Pies, representa una unidad de medida de capacidad inexacta del transporte marítimo (Buques portacontenedores y terminales portuarios para contenedores) expresada en contenedores. 
Los principales servicios se centran en el cargue, descargue, repesaje y movilización de contenedores para inspección y llenado, entre otras. Estas características, junto con los procesos de transformación tecnológicos implantados los consolidan al puerto de Cartagena como el centro logístico integrado y de trasbordo internacional para el Caribe, entre los 100 mejores puertos del mundo, contemplando una inversión de mil millones de dólares, con el fin de duplicar los contenedores que en la actualidad mueve, es decir, mover 5.000.000 contenedores a futuro (Bejarano, 2013).

En el sector logístico portuario de Cartagena se están realizando una serie de inversiones en programas y proyectos que mejoren la capacidad instalada del sector a futuro, es importante mencionar que, según el Consejo Privado de Competitividad, uno de los grandes inconvenientes de Colombia, que lo imposibilitan a entrar adecuadamente en los procesos de integración económica, son los cuellos de botella en infraestructura portuaria, resultados del retraso en el transporte de carga y la cadena logística que repercuten en la competitividad de los productos nacionales, e incluso por debajo de algunos países de la región (Rodríguez, 2013).

También se resalta que en el sector logístico portuario en Colombia no se han implementado políticas y planes que busquen la articulación de los principales puertos nacionales con otros nodos de transporte (multimodal). A este aspecto se le suma la debilidad de las instituciones encargadas de las decisiones estratégicas y de la gestión de procesos de contratación, estas últimas presentan fallas, errores en las asignaciones de las concesión y problemas de corrupción. A su vez, están los problemas relacionados con la tramitología e inspecciones en los puertos, ya que en el país no se cuenta con una sistematización eficiente en los procesos de embarque y descargue de las mercancías (Rodríguez, 2013).

Según el Informe de Competitividad Global 2015-2016, Colombia ocupó la posición 61 de 140 economías evaluadas. En términos de transporte y logística portuaria ocupó el puesto 85 , con una puntuación de 3,6 , por encima del indicador de competitividad de calidad de caminos Ediciones EFIM (posición 126, con 2,7 en calificación final), y en materia de infraestructura el país se ubicó en la posición 84 , con una puntuación de 3,67 , por debajo de países de la Región, como Chile (posición 45), Ecuador (posición 67), y Brasil (posición 74), entre otros (World Economic Forum, 2015-2016).

En términos de ambiente de negocios, lo cual es un buen indicador de competitividad, el informe Doing Business del grupo del Banco Mundial 2015-2016, arrojó resultados no tan positivos en esta materia. En el informe Colombia se ubicó en el puesto 54, afectado de manera implícita por los bajos indicadores en materia logística portuaria, como son los altos costos logísticos en exportación (USD 2.225) e importación (U\$D 2.830) de mercancías por contenedor unitario (World Bank Group, 2016).

Los problemas en la cadena logística portuaria en Colombia impiden la competitividad internacional de los productos nacionales, reprimiendo el aumento de las exportaciones y de la producción nacional. Los principales inconvenientes del sector portuario en Cartagena son los relacionados con la movilidad en el corredor de carga, demoras en la construcción del canal El Varadero, y los relacionados con la sedimentación, quizás uno de los más graves, ya que dichos sedimentos restan profundidad al puerto, lo que interviene en su eficiencia (Rodríguez, 2013). "En Colombia no se puede desconocer la trascendencia que desde tiempos de la época de la colonia han tenidos los puertos como requisito de competitividad y de expansión de poder comercial" (PNUD, 2014, p.16).

Este artículo de investigación tiene como propósito analizar el sector portuario y logístico de la ciudad de Cartagena de Indias, empleando para ello el análisis DOFA como herramienta metodológica.

\section{Metodología}

Esta investigación es de tipo descriptiva y analítica, con información documental basada en fuentes secundarias puesto que se analiza el 
sector logístico portuario en la ciudad de Cartagena, y de igual forma se identifican los principales retos en materia competitiva del sector durante los próximos años. El análisis DOFA es un análisis de vulnerabilidad que se utiliza para medir y determinar el actuar de una empresa o conjunto de empresas, ante una situación que le esté afectando. En otras palabras, es una herramienta diagnostica que muestra el panorama general de la empresa o conjunto de empresas, dentro del medio en el cual se deben mover (Amaya, 2010).

\section{Figura 1. Escuelas de Estrategias de Mint- berg (1998).}

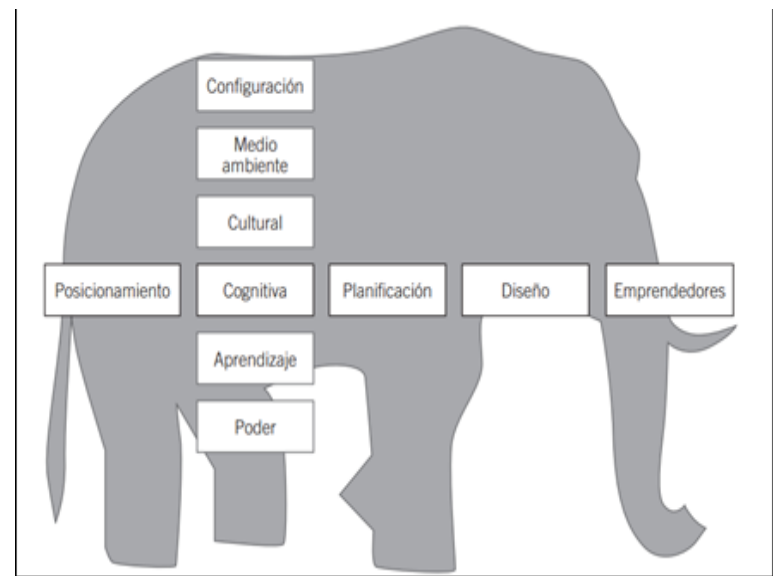

Fuente: Tomado de Rivera \& Malaver (2011)

De acuerdo a Selznick (1957), como pionero de la Escuela del Diseño plantea la estrategia como proceso conceptual, lo cual conlleva a un análisis de situaciones que incluyen el estudio de debilidades, amenazas, fortalezas y oportunidades. Andrews (1971), lo define como el método de identificación de los cuatro aspectos más importantes para una empresa o sector. Donde apunta que el componente de; Fortaleza demuestra las competencias de la empresa y con los que puede tener total control durante su desempeño; Debilidades se refiere a las carencias o los aspectos vulnerables de la empresa y que se traduce en una desventaja dentro del mercado; Oportunidades integra los elementos potenciales a nivel político, económico, social, etc., que pueden ser aprovechados por la empresa con el fin de lograr los objetivos trazados; Amenazas compone aquellos elementos de carácter adversos que ponen en riesgo el logro de los objetivos trazados por la empresa, la duración de estas amenazas son de mediano y largo plazo y se presentan en cualquier momento llevando la situación de la empresa a un estado de incertidumbre.

En sí, el método DOFA se estudia para analizar problemas técnicos de un sector o áreas distintas para las que fue creada inicialmente. Se aplica adecuadamente a procesos de planta, logística, penetración de mercados, preparación de portafolios de inversión empresarial, aplicación de políticas interna, entre otras, los cual, le permite tener a la empresa una poderosa herramienta de información para la toma de decisiones en temas de gran impacto (Amaya, 2010) En otras palabras, el fin último del análisis DOFA es:

(...) la planeación estratégica que lleve a la empresa a integrar procesos que se anticipen o minimicen las amenazas del medio, el fortalecimiento de las debilidades de la empresa, el potenciamiento de las fortalezas internas y el real aprovechamiento de las oportunidades. El resultado es un plan de trabajo conjunto e integrado a todo nivel de la empresa, de tal manera que todas las actividades y compromisos se complementen para que todos los esfuerzos de la compañía vayan en un solo sentido. (Amaya, 2016, p.3)

La importancia del análisis DOFA en la planeación estratégica permite a una empresa o sector acercarse a la visualización y construcción de su futuro, ya que le permite determinar los propósitos y estrategias que orientan la adquisición, uso, control y eficiencia de los recursos, para realizar o conseguir dichos propósitos. Adicionalmente, la utilización de este método permite a la empresa o al sector que se esté estudiando, proponer acciones y estrategias, las cuales surgen de un proceso de análisis y concatenación de recursos y fines, que conlleven a la competitividad de la misma, entendida la competitividad con la capacidad que tiene la empresa o el sector de crear bienes o servicios con valor agregado que le permitan conservar e incrementar posición en el mercado frente a sus competidores (Ramírez-Rojas, 2009).

En términos más específicos, este método consiste en la identificación de cuatro aspectos imAnfibios ISSN: 2665-1513 
portantes para la empresa o sector; Fortalezas, Debilidades, Oportunidades y Amenazas. El primer componente, hace referencia aquello en lo que la empresa o sector es competente. Se traduce en aquellos elementos que están bajo su control, mantienen un alto nivel de desempeño, los cuales generan ventajas en el presente y en el futuro. Las principales fortalezas se evidencian en excelente y eficiente recurso humano, con capacidades y destrezas para hacer las actividades, sistemas de trabajo eficiente, costos bajos, productos y servicios competitivos, imagen institucional reconocida, convenios y asociaciones con otras empresas (Amaya, 2010; Ponce, 2007; Ramírez-Rojas, 2009).

En cuanto a las Debilidades, este componente hace referencia a las carencias o deficiencias. Son aquellos elementos, en los que la empresa o el sector tienen bajos niveles de desempeño y por tanto es vulnerable. A su vez, este componente denota una desventaja, ante la competencia, con posibilidades poco eficientes en el futuro, es decir constituyen un obstáculo para la consecución de los objetivos. Al igual que las fortalezas se manifiestan en el recurso humano, tecnología, organización, productos, imagen, entre otros. De igual forma, este componente, junto con las fortalezas, constituyen variables externas de la organización, es decir hacen referencia a los límites determinados del sector productivo y el entorno general que define el ambiente competitivo (Amaya, 2010; Ponce, 2007; Ramírez-Rojas, 2009).

En tercer lugar, las Oportunidades hacen referencias aquellos elementos que son potencialmente favorables para la organización, los cuales son utilizados para alcanzar o superar los objetivos. En este sentido, las oportunidades se pueden presentar en cualquier ámbito; político, económico, social, tecnológico, cultural, etc., dependiendo de la naturaleza de la organización o el tipo de sector que se esté analizando. En últimas, la importancia de este componente está, en que, su reconocimiento representa un reto para los directivos, ya que no se pueden crear ni adaptar estrategias sin identificar y evaluar antes el potencial de crecimiento y utilidades de cada una de las oportunidades prometedoras o potencialmente importantes (Amaya, 2010; Ponce, 2007; Ramírez-Rojas, 2009).

Ediciones EFIM
Por último, el componente Amenazas, hace referencia a todos los elementos del entorno que surgen en circunstancias adversas, las cuales ponen en riesgo la consecución de los objetivos establecidos. Por lo general, las amenazas se presentan repentinamente o de manera prolongada, creando una condición de incertidumbre e inestabilidad, en las cuales la empresa o el sector no tienen gran influencia. Por ejemplo, en cuanto a la tecnología, competencia agresiva, productos nuevos o más baratos, impuestos, restricciones, inflación, entre otros. En este sentido, resulta de vital importancia para la empresa o sector, reconocer de manera oportuna aquellas situaciones que signifiquen riego en su competitividad o su posición futura (Amaya, 2010; Ponce, 2007; Ramírez-Rojas, 2009) (Ponce, 2007) (Ver Figura 2).

\section{Figura 2. Matriz conceptual DOFA}

\begin{tabular}{|l|l|}
\hline \multicolumn{2}{|c|}{ FORTALEZAS } \\
\hline Capacidades fundamentales en áreas cla- & No hay una dirección estratégica clara. \\
ves. & Instalaciones obsoletas. \\
Recursos financieros adecuados. & Rentabilidad inferior al promedio. \\
Buena imagen de los compradores. & Falta de oportunidad y talento gerencial. \\
Ser un reconocido lider en el mercado. & Seguimiento deficiente al implantar la \\
Estrategias de las áreas funcionales bien & estrategia. \\
ideadas. & Abundancia de problemas operativos in- \\
Acceso a economías de escala. & ternos. \\
Aislada (por lo menos hasta cierto grado) & Atraso en investigación y desarrollo. \\
de las fuertes presiones competitivas. & Linea de productos demasiado limitada. \\
Propiedad de la tecnologia. & Débil imagen en el mercado. \\
Ventajas en costos. & Débil red de distribución. \\
Mejores campanas de publicidad. & Habilidades de mercadotecnia por debajo \\
Habilidades para la innovación de productos. & del promedio. \\
Dirección capaz. & Incapacidad de financiar los cambios ne- \\
Posición ventajosa en la curva de expe- & cesarios en la estrategia. \\
riencia. & Costos unitarios generales más altos en \\
Mejor capacidad de fabricación. & relación con los competidores clave. \\
Habilidades tecnológicas superiores. & \\
\hline
\end{tabular}

\begin{tabular}{|l|l|}
\hline \multicolumn{1}{|c|}{ OPORTUNIDADES } & \multicolumn{1}{c|}{ AMENAZAS } \\
\hline Atender a grupos adicionales de clientes. \\
Ingresar en nuevos mercados o segmentos. & Entrada de competidores foráneos con \\
Expandir la línea de productos para satis- & costos menores. \\
facer una gama mayor de necesidades de & Incremento en las ventas y productos sus- \\
los clientes. & titutos. \\
Diversificarse en productos relacionados. & Crecimiento más lento en el mercado. \\
Integración vertical (hacia adelante o hacia & Cambios adversos en los tipos de cambio y \\
atrás). & politicas comerciales de gobiernos extran- \\
Eliminación de barreras comerciales en & jeros. \\
mercados foráneos atractivos. & Requisitos reglamentarios costosos. \\
Complacencia entre las compañias rivales. & Vulnerabilidad a la recesión y ciclo empre- \\
Crecimiento más rápido en el mercado. & sarial. \\
& Creciente poder de negociación de clientes \\
& o proveedores. \\
& Cambio en las necesidades y gustos de los \\
& compradores. \\
& Cambios demográficos adversos. \\
\hline
\end{tabular}

Fuente: Thompson y Strikland (1998), como se citó en Ponce, (2007, p.116) 


\section{Resultados}

\subsection{Análisis DOFA del sector logístico portuario de la ciudad de Cartagena}

Actualmente el comercio global ha crecido más rápido que el producto interno bruto, lo que ha conllevado a cambios considerables en la infraestructura y en las estrategias comerciales que adoptan los países, pero con mayor intensidad en la parte logística portuaria, ya que este sector es fundamental en las relaciones comerciales entre un país y otro. Este es el caso de Colombia, el cual ha experimentado cambios en su logística portuaria, dado los cambios y los tratados de libre comercio que ha firmado con algunos países y las relaciones que cada día va forjando. Ahora bien, estos cambios no se han dado en todas las ciudades del país, ya que no todas cuentan con puerto u otro mecanismo que facilite el comercio.

En este contexto, Cartagena resulta ser una de esas ciudades, afortunadas por contar con puerto, en uno de los lugares más estratégicos de la región e internacionalmente. Sin embargo, al igual que otros sectores económicos presenta debilidades, amenazas, fortalezas y oportunidades, las cuales son de gran interés para el desarrollo de esta investigación. Por ello, el desarrollo de este capítulo se centra en identificar estos cuatro componentes del sector, pero antes se realiza una descripción general del sector logístico portuario. Cabe resaltar que la mayor parte de la información con la que se desarrolla este capítulo, fue obtenida de la página web de la Sociedad Portuaria Regional de Cartagena, principalmente de cada una de sus revistas, edición 2007, 3008, 2009, 2010, 2011, 2014, entre otras.

Con la crisis económica de 2008 , la región latinoamericana sufrió una serie de altercados, uno de ellos fue la disminución de la inversión en puertos, ya que dicha recesión implicó carencia de recursos para invertir en este sector. Colombia y Panamá, fueron dos casos a resaltar; debido a la recesión fueron países que permanecieron constantes en la inversión logística portuaria, e incluso teniendo en cuenta la incertidumbre que ello representó en movimientos de carga, dado que en el futuro no sabían con certeza si barcos más grandes elegirían sus puertos como lugar de destino.

El sector logístico portuario de Cartagena, dentro de sus empresas más importantes como la Sociedad Portuaria Regional de Cartagena (SPRC) ha venido realizando una gran inversión, teniendo en cuenta las proyecciones de la carga a recibir con la ampliación del Canal de Panamá y con las inversiones en materia portuaria que se están reiniciando en Latinoamérica (Costa Rica, Perú, México, Guatemala). Esto con el fin de mantener y fortalecer la ventaja comparativa, pero con mayor énfasis en romper con el paradigma del comercio tradicional, que es exportar materia prima a los países industrializados e importar productos terminados de dichos países. Y, por otra parte, darle paso al comercio entre países latinoamericanos, con el propósito de lograr autosuficiencia comercial; propiciando a nuevos servicios marítimos, que implícitamente traen alimentadores, ferrys, barcazas, entre otras naves no tradicionalistas.

La SPRC con miras en cumplir con su visión a 2020, tuvo en cuenta el potencial de Colombia en materia exportadora (exportaciones e importaciones, no solo estar atenido al trasbordo), para poner en marcha distintos programas y planes de inversión en tecnología moderna como: adquisición de grúas modernas especializadas en el manejo de contenedores, multiplicó su capacidad, aumentó la capacidad de su muelle y profundizó las zonas adyacentes, figurando como una de las mejores estrategias del sector y como respaldo a las inversiones que realizó y que se están realizando. De igual forma, tuvo en cuenta el crecimiento económico que han registrado la mayor parte de los países de la región en los últimos cinco años.

Por otra parte, la ampliación del Canal de Panamá, como se mencionó anteriormente es una de las apuestas más importantes para el sector logístico portuario de la ciudad. Este proyecto aprobado en 2006, ha sido y será por muchos años un hito en la historia de la ingeniería y el sector portuario. La razón de ser de este mega proyecto fue unir un océano con el otro, con el fin de conservar su importancia como canal Anfibios ISSN: 2665-1513 
interoceánico, el cual había estado subestimado, dado que, algunos contenedores tuvieron que tomar la ruta del sur para poder pasar de un océano a otro, porque las dos esclusas con las que contaban no tenían la capacidad, a consecuencia de esto, se deriva el nombre de buques Post Panamax. Ahora bien, con la ampliación, se espera que los ingresos del puerto de Panamá se aumentaran nueve veces, algo que es considerablemente lógico, ya que uno de los buques de la nueva generación equivale en carga a lo que pueden contener 570 aviones o 5.800 camiones, los cuales, sumados, tendrían una extensión de 97 kilómetros de largo.

Ahora bien, La SPRC en representación del Puerto de Cartagena, ha podido identificar su participación en el sector logístico portuario con la ampliación de este canal, aumentando y adecuando la infraestructura con la que cuenta (más de 500 millones de dólares), como lo son Contecar y el Muelle de Manga, es decir que cuenta con una de las principales plataformas logísticas del continente. Por otra parte, la SPRC tuvo en cuenta los patrones de comercio que se aproximan (2020), en los cuales las flotas marinas y los mercados internacionales movilizaran portacontenedores en dirección al eje ecuatorial Este-Oeste, y estar el puerto de Cartagena en medio de esta línea, necesariamente requería una restructuración.

En este sentido, algunas empresas del sector logístico portuario de Cartagena como SPRC y CONTECAR, en su Plan Maestro de Desarrollo, designaron inversiones de más de \$290 millones de dólares en los terminales. Este proceso se inició con el dragado de sus dársenas y la paliación de sus muelles marginados en Contecar, los cuales equivalen a $700 \mathrm{mts}$, al igual que en la SPRC, los cuales equivalen a $700 \mathrm{mts}$. Adicionalmente, se realizaron inversiones en la consecución de cinco Grúas Pórtico Súper Pos Panamax, con alta calidad y productividad. Dentro de este mismo proceso, se adecuaron 15 hectáreas de patíos, con las cuales se completaron 27 en total en Contecar y en la SPRC otras cuatro. A su vez, adquirieron 24 Grúas RTGs, de las cuales, 10 pasaron a la SPRC y 14 a Contecar, esta maquinaria fue adquirida con el fin de ofrecer mayor eficiencia, robustez y preciEdiciones EFIM sión con los sistemas de información instalados en ambos terminales.

En este sentido, en el puerto se destinaron recursos importantes para incrementar la oferta de servicios logísticos, construyendo la primera fase del centro de distribución ubicada en Contecar, la cual hace referencia a una bodega de 20 metros de altura y 70 de ancho, con un área de $10.000 \mathrm{~m} 2$, cuenta con 32 muelles de carga, puertas eléctricas y niveladoras para facilitar el acceso a esta misma. La razón de ser de esta primera fase es poder almacenar más de 18.000 pallets, entre otras cosas.

Así las cosas, Cartagena se ha convertido en un puerto misceláneo, el cual, a partir de 2006 con el dragado del canal de acceso a la bahía, con profundidad de 15 metros, tiene cabida para recibir gránelos sólidos, gránelos líquidos, carga suelta general y cruceros turísticos, entre otros, con lo cual, se espera que, en 2020, la movilización de carga supere los 15.000 TEUs. Esta situación a su vez, se traduce en un importante rubro económico para el país, principalmente por los fletes obtenidos, incrementando la competitividad y vocación exportadora de muchos de los productos que se producen en el interior. Cada uno de estos aspectos contribuye a que el puerto de Cartagena, esté entre los puertos más importantes del mundo, catalogado como mejor puerto del Caribe en cinco oportunidades por la Caribbean Shipping Asociatión. Adicionalmente, ha sido uno de los primeros puertos del continente en recibir la certificación de CTQI (Container Terminal Quality Indicators), y posteriormente la certificación de la ISO 9001:2008, lo que indica que sus procesos operativos, técnicos y administrativos ofrecen garantía total para sus clientes y la sociedad.

Desde la perspectiva social, el puerto le apunta al mejoramiento y al desarrollo de la ciudad. En promedio en los últimos cinco años, el puerto abarcó más de 5.000 empleos, contribuyendo al desarrollo y sostenibilidad de la ciudad. Por otra parte, el puerto de conecta con más de 600 puertos en 140 países, lo que significa conectividad regional en concepto de tiempo y distancia, traducidos en menores fletes, incentivo fundamental para los empresarios, mejoras en 
Diógenes Alejandro Arrieta Rodríguez, Diego A. Cardona A.

la competitividad y por ende aumentos del PIB departamental y nacional.

También es preciso y pertinente mencionar que, en Contecar en 2011, se movilizaron 40.000 vehículos, debido a esto la organización construyó una terminal marítima especializada en el movimiento de carga autopropulsada. También es de resaltar el muelle flotante (160 metros) que se construyó en dicha terminal, especializado en la atención de naves Ro-Ro y 12 hectáreas destinadas al almacenamiento, también cuenta con un servicio operativo para maximizar los recursos y automatizar todos los procesos relacionados con los vehículos, a este proyecto fue al que se le llamó el HUB de automóviles del Caribe. A su vez, este sistema ofrece otros servicios como el lavado, pintura, toma de improntas, adición de accesorios, entre otros, lo cual permiten darle valor agregado a los servicios que ofrece. Actualmente, este sistema le ha permitido al puerto ser el principal terminal y centro de distribución de General Motors y Dealers (Chrysler), al igual que la exportación de vehículos al Caribe, México y Brasil. A su vez, contribuye al desarrollo de ensambladoras y el mercado automotor del país, dada la creciente demanda de automóviles en Colombia.

Según las proyecciones del Banco Mundial, a 2020, los costos del transporte de mercancías disminuirán en un $50 \%$, lo que representa una gran oportunidad para el Puerto, tanto así, que las proyecciones de la Institución prevén que aumenten hasta cinco veces el movimiento mercantil entre un país y sus socios. A su vez, las estadísticas prevén que el HUB del Caribe movilizará 4,5 millones de TEUs, lo que disminuirá los costos de transacciones, esto a su vez se traduce en un componente esencial para el aumento del PIB departamental y nacional.

Por otra parte, las estadísticas económicas proyectan que en los próximos años se dará una conexión comercial significativa entre Colombia y Brasil, los cuales actualmente no tienen una gran ni tampoco trascendental relación comercial, pero se prevé que la inversión extranjera directa de Brasil en Colombia aumente, especialmente en el sector portuario, ya que Brasil se ha posicionado como la octava mayor economía global, y es uno de los países con mayor con- sumo en Sur América. También es de resaltar que las políticas de desarrollo económico implementadas en Brasil sobre los territorios marginados del norte, representa una gran ventaja comercial para el puerto, ya que esta región es potencialmente consumidora de productos colombianos, principalmente los que tiene que ver con la agroindustria, manufactura, sector textil y servicios. En otras palabras, Brasil representa un mercado potencial relevante para Colombia, ya que cuenta con más de 40 millones de habitantes de clase media, con un fuerte rezago en infraestructura portuaria, permitiéndole a Colombia, a través del puerto de Cartagena, atender de manera eficiente los cinco puertos de Brasil. Así, si es de cumplirse con todas estas expectativas, el puerto de Cartagena realizará un papel sumamente importante en esta creciente demanda del país vecino.

Con base en lo anterior y como complemento, resulta pertinente evaluar cómo ha sido el comportamiento de las principales estadísticas del Puerto en el tiempo, para tal fin a continuación, se hace un breve análisis de los principales indicadores del Puerto de 2000 a 2015. Para lograrlo se tuvo información de indicadores básicos como: recaladas de motonaves, toneladas movilizadas por tipo de estiba, movimiento de carga contenerizada (TEUs), entre otras.

En la Figura 4, se puede observar el comportamiento ascendente que ha tenido las recaladas de motonaves en los últimos 15 años, el cual tuvo un comportamiento promedio de 1.866 recaladas. De la Figura en mención, se pueden rescatar tres grandes hallazgos; un primero hace referencia a que, a partir de 2006 con la iniciativa y puesta en marcha de Contecar, la movilización de este componente aumentó significativamente, tanto así que de 2005 a 2006, aumentó en un 35\% aproximadamente. A partir de este año (2006), el ritmo de crecimiento ha sido exponencial, registrando el nivel más alto en 2015, y se espera que para 2020, duplique los niveles observados de 2009. Un segundo hallazgo, hace mención a que el ritmo de crecimiento de este indicador, teniendo en cuenta los niveles de 2000 y 2015 , fue de $143,4 \%$ cifra extraordinaria, pues cabe resaltar que, en el 2008, se Anfibios ISSN: 2665-1513 
presentó una importante recesión económica, la cual no repercutió significativamente en el comportamiento de este indicador. Un tercer hallazgo es que, de este total de recaladas, el $64 \%$ son de trasbordo y un $36 \%$ son descargue y cargue doméstico.

Figura 3. Recaladas de Motonaves anuales en la Sociedad Portuaria Regional de Cartagena

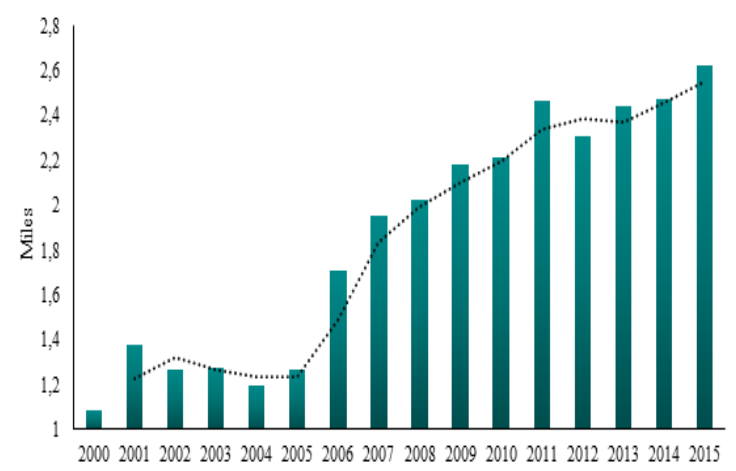

Fuente: elaboración propia con base en Organización Puerto de Cartagena

Por otra parte, las toneladas movilizadas en la SPRC, se pueden desagregar en cuatro componentes para hacer más enriquecedor el análisis. En primer lugar, se puede observar en la Figura 5, el comportamiento de las toneladas movilizadas según el tipo de estiba: Descargado Domestico, el cual presentó un promedio de 2.023.515 toneladas movilizadas. De este total el $92 \%$ corresponde descarga de contenedores, un $6 \%$ a carga general y el resto $(2 \%)$ a granel. De la Figura 5, también se puede apreciar el comportamiento tan variado en la movilización de toneladas de descargue de mercancía general. Solo en 2006, y en 2008 presentaron alces en su comportamiento, de 2009 en adelante ha tendido a decrecer. Por su parte, el descargue de contenedores a medida que pasan los años, se observa un comportamiento ascendente, por lo cual se puede inferior que en los años venideros este rubro será mucho mayor. En términos generales, el promedio de carga descargada en contenedores ha sido de 1.853 .759 , con un ritmo de crecimiento anualizado del $10 \%$.
Figura 4. Toneladas movilizadas por tipo de estiba; Descargado doméstico, 2000-2015

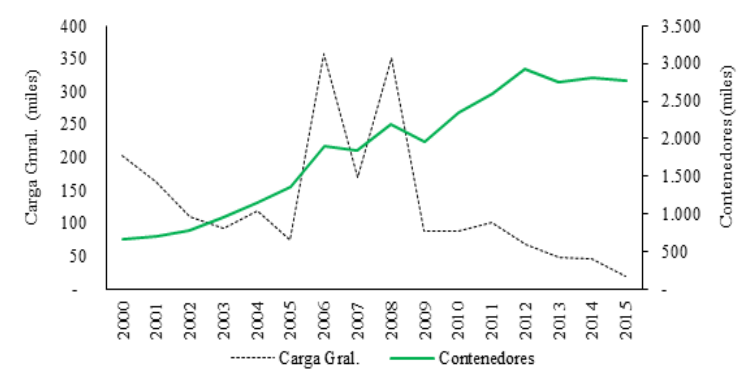

Fuente: elaboración propia con base en Organización Puerto de Cartagena

En termino de cargue doméstico, en la Figura 6 se puede observar que el comportamiento de este rubro ha sido principalmente por contenedores. De 2000 a 2015, el promedio de toneladas cargadas ha sido de 1.646.669 toneladas, de las cuales el $98,5 \%$ han sido en contenedores, el $1,4 \%$ en carga general y el resto $(0,11 \%)$ de granel. Se pude evidenciar en la Figura en mención que, el año donde hubo mayor carga de mercancía general fue en 2007, y en niveles inferiores 2011 y 2014. Por su parte, el cargue mayor de contenedores se realizó en 2014.

Figura 5. Toneladas movilizadas por tipo de estiba; Cargado doméstico, 2000-2015

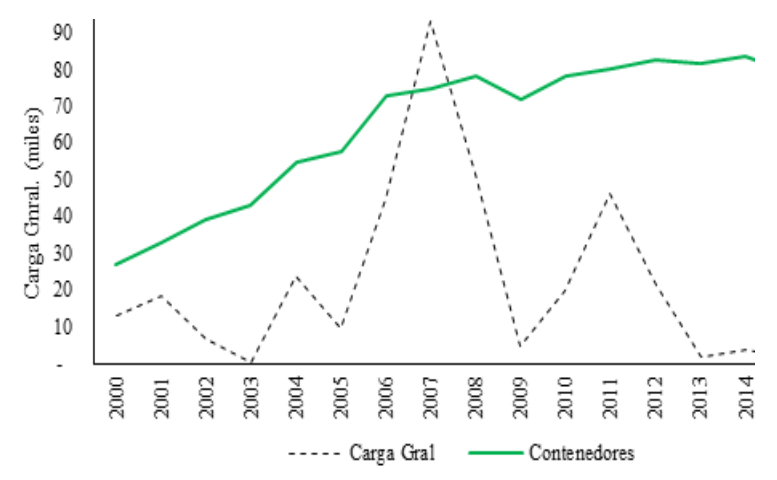

Fuente: elaboración propia con base en Organización Puerto de Cartagena

Por otra parte, se tiene el comportamiento de la mercancía para trasbordo, la cual se subdivide en mercancía descargada y cargada. En la Figura 7 , se puede observar el comportamiento de los principales rubros de descarga de trasbordo en la SPRC de 2000 a 2015. De la gráfica se desprenden tres aspectos muy importantes; un primero es que, el crecimiento de contenedores 
descargados para trasbordo tiene un crecimiento, casi que exponencial. Por su parte el descargue de mercancía general tiene un comportamiento muy variado, presentando altos en los años 2006, 2012, y 2014, en los demás años, el comportamiento ha sido decreciente. Un segundo aspecto a resaltar es que, de 2006 en adelante, al igual que la mayoría de los demás indicadores, el descargue de contenedores para trasbordo ha sido mucho mayor. Por último, se evidencia que el promedio de toneladas descargada para trasbordo en estos quince últimos años fue de 3.784 .870 toneladas con un promedio de crecimiento anualizado de $15 \%$.

Figura 6. Toneladas movilizadas por tipo de estiba; Descargado Trasbordo, 2000-2015

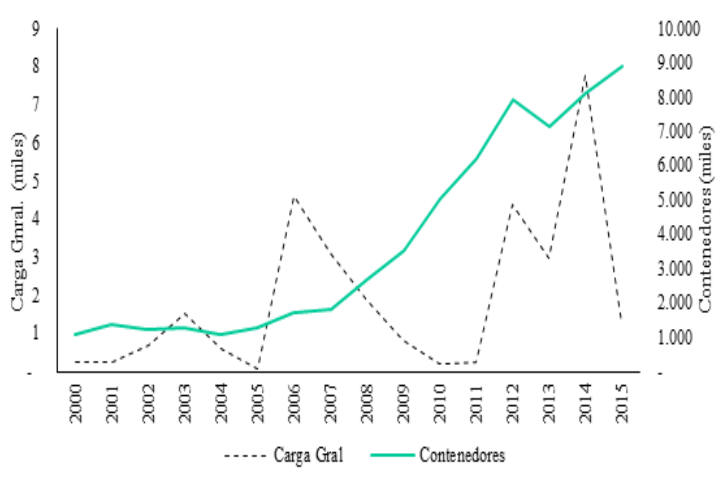

Fuente: elaboración propia con base en Organización Puerto de Cartagena

En este sentido es importante resaltar el comportamiento de la mercancía cargada en el puerto para trasbordo. En la Figura 8, se puede observar que de 2000 a 2006 el cargue de contenedores para trasbordo se mantenía casi que constante, sin embargo, a partir de este último año, el incremento fue considerable, alcanzando un máximo en 2012 y en 2015, y según su tendencia se espera que aumente mucho más en 2020. Por su parte, el cargue de mercancías en general ha sido muy variado, a diferencia del anterior componente, no presenta un patrón definido. Alcanza unos picos en el 2007, posteriormente disminuye e incrementa nuevamente en 2012. Por último, se evidenció que el promedio de cargue de mercancía para trasbordo en los últimos quince años fue de 3.786 .863 toneladas, con un ritmo de crecimiento anualizado de $15 \%$.
Figura 7. Toneladas movilizadas por tipo de estiba; Cargue- Trasbordo, 2000-2015

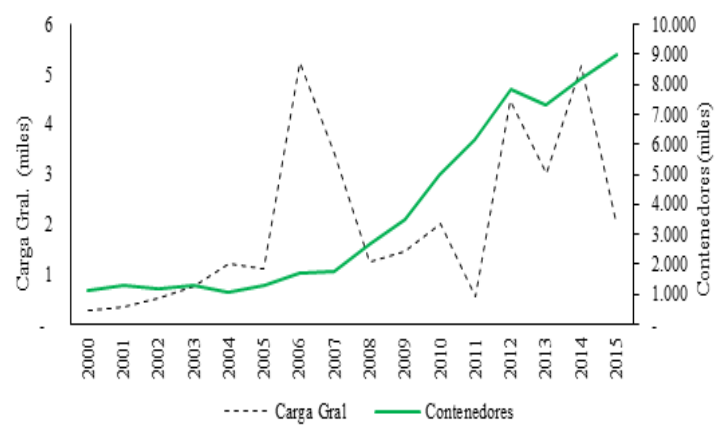

Fuente: elaboración propia con base en Organización Puerto de Cartagena

En términos generales, en la Figura 9 se puede observar el comportamiento del total de toneladas movilizadas en la Sociedad Portuaria Regional de Cartagena. El total de toneladas movilizadas durante estos quince años fue de 179.870.674 toneladas, con un promedio anual de 11.241.917, presentando un crecimiento anualizado de $12,6 \%$. En este total la mayor participación o el mayor número de toneladas movilizadas ha sido por concepto de trasbordo tanto en descargue como cargue. Otro aspecto a resaltar de esta gráfica es el comportamiento ascendente que tiene la movilización de carga en el puerto, es significativo al comparar las cifras entre este intervalo de tiempo. Por ejemplo, en 2000, el total de carga movilizada fue de 3.807.397 toneladas, y para 2015 la cifra pasó a 22.698.318 toneladas, lo que se traduce en un incremento en más de $495 \%$, aumento considerablemente alto.

Figura 8. Total, Toneladas movilizadas por tipo de estiba en la Sociedad Portuaria Regional de Cartagena, 2000-2015

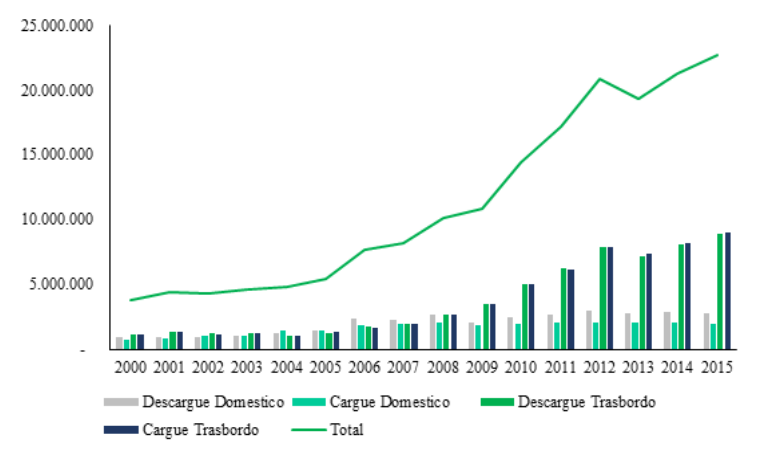

Fuente: elaboración propia con base en Organización Puerto de Cartagena

Anfibios ISSN: 2665-1513 
Por otra parte, es pertinente mencionar el comportamiento que ha tenido la carga movilizada en contenedores (medida en TEUs) en los últimos 20 años. En la Figura 10, se observa el comportamiento ascendente de este componente a partir de 2006. Mientras que en este último año (2006), se movilizaban 744.411 TEUs, en 2015 la cifra pasó a ser de 2.439.307 TEUS, lo que se traduce en un incremento de $228 \%$, con un ritmo de crecimiento promedio anualizado de 23,3\%. En sí, de 1994 a 2015, en la SPRC se han movilizado 19.216.570 TEUS.

Figura 9. Total, Toneladas movilizadas por tipo de estiba en la Sociedad Portuaria Regional de Cartagena, 2000-2015

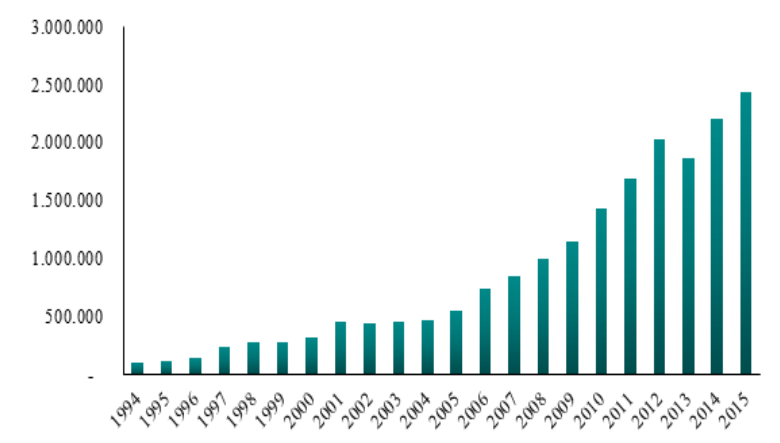

Fuente: elaboración propia con base en Organización Puerto de Cartagena

Con base en cada uno de estos resultados se tienen las bases para entrar con mayor detalle a conocer las debilidades, oportunidades, fortaleza y amenazas de este sector, por ello el siguiente apartado estará dirigido a cumplir con dicho objetivo.

\section{Análisis DOFA del sector logístico portuario de la ciudad de Cartagena}

El análisis estratégico DOFA, parte de evaluar tanto internamente como externamente los factores y determinantes que está expuesto el sector logístico portuario, a su vez permite entender las ventajas y desventajas que tiene. Para ello, en primera instancia, se deben eliminar al máximo las amenazas que se están presentando en el sector por medio de las fortalezas que internamente tiene. Es confortante que haya una estabilidad económica regional y la posible entrada de nuevos países compradores comerciales, de igual forma, el tener un excelente posicionaEdiciones EFIM miento global ganado por el amplio portafolio de productos y servicios que ofrece el Puerto, le permite tomar acciones y respuestas frente a las amenazas que presenta y seguir manteniendo un excelente nivel competitivo.

Por otro lado, el boom tecnológico, la nueva era digital y el creciente interés de las empresas internacionales por hacer uso de nuevas tecnologías portuarias, genera una oportunidad para potencializar las fortalezas que posee el puerto de Cartagena, y lograr mantener su ventaja competitiva. Finalmente, se debe prestar mucha atención a las debilidades, pues se deben mitigar en lo posible y deben convertirse en fortalezas de este sector, con el fin de poder hacerle frente a las amenazas, y también aprovechar las oportunidades que vayan presentándose.

Ahora bien, el problema/oportunidad que se manejará en el presente documento nace de la revisión de las debilidades encontradas en el sector logístico portuario. Principalmente aquellas relacionadas con la organización territorial, ya que en Colombia se presentan dos patrones, es decir, por el lado de las costas se presenta un patrón o están orientadas a comercio exterior, con un fuerte carácter exportador, mientras que en el interior del país se registran los mayores centros de producción y de consumo. A su vez, está el problema relacionado con las grandes distancias por carretera entre los puertos y los principales centros de producción y de consumo del país.

Finalmente, se encontró que presenta problemas de movilidad con el corredor de carga, a pesar que está mejores condiciones que el de Barranquilla, presenta inconvenientes para atender el aumento del flujo de entrada y salida de mercancías que se están presentando en el puerto, es decir no cuenta con una vía alterna que descongestione dicho tráfico, e incluso, en ocasiones afecta otras vías de la ciudad, principalmente las del norte. Entre otras cosas, presenta inconvenientes por el retraso en la construcción del canal el Varadero, ya que con este canal se podría ampliar el canal del Dique, para poder responder al incremento de la demanda del puerto por la ampliación del Canal de Panamá. 
Por otra parte, como fortaleza se tiene una fuerza de movilización de carga excelente, caracterizada por tener buena planeación logística y bajos costos. Ahora bien, como oportunidad se evidencia que son cada vez más los mercados que se están abriendo al mundo, permitiendo los tratados de libre comercio entre países, de igual forma se prevé el aumento de la demanda de servicios con ampliación del Canal de Panamá, al igual que el aumento de la demanda de productos por parte de Brasil, uno de los países más prósperos de la región, entre otras, las cuales resultan oportunidades ventajosas en materia competitiva y por ende se deben aplicar estrategias que permitan mantenerlas; para así poder obtener mejores resultados y ser cada vez más competitivos. A continuación, se presenta de manera más detallada las debilidades, oportunidad, fortalezas y amenazas del sector logístico portuario en la ciudad de Cartagena.

Uno de las debilidades que pueden convertirse en oportunidades para el Puerto es la falta de infraestructura vial en el país. Este problema histórico, ocasiona fuertes consecuencias para el comercio exterior, ya que más del $50 \%$ de las empresas colombianas se encuentran ubicadas en el centro del país, generando problemas logísticos para el transporte de mercancías con destino a la exportación. Sin embargo, con la implementación de estrategias logísticas que permitan que a través del Río Magdalena se puedan transportar a menor costo y tiempo la mercancía del centro del país hasta el puerto de Cartagena, se podrían articular en mayor medida la cadena logística y de exportación de muchos productos nacionales que actualmente no tiene la capacidad o medios para exportarlos.

\section{Tabla 1. Análisis DOFA del sector Logístico Portuario de Cartagena}

\begin{tabular}{|c|c|}
\hline Debilidades & Fortalezas \\
\hline \multirow{2}{*}{$\begin{array}{l}\text { Presenta rezago en materia de avan- } \\
\text { ce físico y tecnológico, en compa- } \\
\text { ración con puertos internacionales. }\end{array}$} & Aprovechamiento de los recursos existentes: las vías terrestres y fluviales. \\
\hline & Capacitación continua y la adquisición de nuevas tecnologías. \\
\hline \multirow{2}{*}{$\begin{array}{l}\text { No es integral en cada uno de sus } \\
\text { eslabones, es decir, es decir no } \\
\text { cuenta con vías alternas necesarias } \\
\text { de transporte. }\end{array}$} & $\begin{array}{l}\text { Capacitación del talento humano; factor fundamental para que la inversión sea en un } 100 \% \text { eficiente y tenga } \\
\text { buenos retornos }\end{array}$ \\
\hline & Eficiencia en la estructura administrativa del sector. \\
\hline $\begin{array}{l}\text { No está haciendo uso de su máxima } \\
\text { capacidad instalada. }\end{array}$ & Está conectado con más de 600 puertos en 140 países y principales líneas navieras a nivel mundial \\
\hline $\begin{array}{l}\text { El número de empleados es inferior } \\
\text { al registrado en puertos internacio- } \\
\text { nales de igual magnitud. }\end{array}$ & $\begin{array}{l}\text { Cuenta con } 51 \text { concesiones lo cual permite que existan empresas especializadas en diferentes servicios brin- } \\
\text { dándole total versatilidad frente a los demás sectores nacional e internacionales }\end{array}$ \\
\hline \multirow{2}{*}{$\begin{array}{l}\text { El calado manejado tiene ( } 45 \text { pies), } \\
\text { está por encima del puerto de Ba- } \\
\text { rranquilla ( } 36 \text { pies), pero está por } \\
\text { debajo del puerto de Miami ( } 50 \\
\text { pies). }\end{array}$} & Cuenta con una variedad de clientes provenientes de Asia, Europa y América \\
\hline & Inversión en mejora y mantenimiento; mejorando los tiempos de transporte \\
\hline \multirow{2}{*}{$\begin{array}{l}\text { La longitud del puerto }(2.058 \mathrm{~m}) \text { es } \\
\text { competitivo frente al de Barranqui- } \\
\text { lla }(1.698 \mathrm{~m}) \text {, pero es inferior al de } \\
\text { Miami }(2.096 .346 \mathrm{mts} 2)\end{array}$} & $\begin{array}{l}\text { Es uno de los puertos más importantes de Colombia y se perfila como el más atractivo para empresas tanto } \\
\text { nacionales como internacionales. }\end{array}$ \\
\hline & $\begin{array}{l}\text { Cercanía con el Canal del Dique, cercanía con el río Magdalena, contribuyendo así, a la navegación y transpor- } \\
\text { te de mercancías hacia otras plataformas. }\end{array}$ \\
\hline \multirow{2}{*}{$\begin{array}{l}\text { Problemas de movilidad con el co- } \\
\text { rredor de carga. No cuenta con una } \\
\text { vía alterna que descongestione di- } \\
\text { cho tráfico, e incluso, en ocasiones } \\
\text { afecta otras vías de la ciudad, prin- } \\
\text { cipalmente las del norte. }\end{array}$} & $\begin{array}{l}\text { Las aguas que lo rodena se mantiene en calma durante casi todo el año, lo que hace posible y seguro las em- } \\
\text { barcaciones que llegan. }\end{array}$ \\
\hline & $\begin{array}{l}\text { Es uno de los puertos más avanzados en tecnología y modernización del país, al contar con equipos apropiados } \\
\text { para el manejo de contenedores, en los últimos seis años }\end{array}$ \\
\hline \multirow{2}{*}{$\begin{array}{l}\text { Presenta inconvenientes en la cons- } \\
\text { trucción del canal el Varadero, ya } \\
\text { que con este canal se podría am- } \\
\text { pliar el canal del Dique, para poder } \\
\text { responder al incremento de la de- } \\
\text { manda del puerto por la ampliación } \\
\text { de Panamá. }\end{array}$} & $\begin{array}{l}\text { Cuenta con los servicios de cargue y descargue de contenedores, es decir desplazamiento de la carga entre los } \\
\text { módulos del puerto. Al igual repasaje de contenedores. }\end{array}$ \\
\hline & $\begin{array}{l}\text { La mayor parte de las empresas cuentan con la movilización de los contenedores para inspección y moviliza- } \\
\text { ción de estos para llenado. }\end{array}$ \\
\hline
\end{tabular}




\begin{tabular}{|c|c|}
\hline Debilidades & Fortalezas \\
\hline \multirow{2}{*}{$\begin{array}{l}\text { Según el Informe de Competiti- } \\
\text { vidad Global (ICG) 2015-2016, } \\
\text { Colombia ocupó la posición } 85 \text { en } \\
\text { términos de transporte y logística } \\
\text { portuaria ( } 140 \text { economías evalua- } \\
\text { das), por encima solamente del in- } \\
\text { dicador de competitividad calidad } \\
\text { de caminos (posición 126). }\end{array}$} & $\begin{array}{l}\text { Cuenta con los tres muelles más importantes de la región; Terminales de Contecar (Terminal de Contenedores } \\
\text { de Cartagena), Muelles el Bosque y la zona industrial de Mamonal. }\end{array}$ \\
\hline & $\begin{array}{l}\text { Ofrece } 11 \text { sitios de atraque especializados en atender a los barcos de carga, con capacidad de atender barcos } \\
\text { hasta de } 5.500 \text { TEUs. }\end{array}$ \\
\hline \multirow[b]{2}{*}{$\begin{array}{l}\text { Según el Doing Business del gru- } \\
\text { po del Banco Mundial 2015-2016, } \\
\text { arrojó resultados no tan positivos } \\
\text { en esta materia. Colombia se ubicó } \\
\text { en el puesto 54, afectado de manera } \\
\text { implícita por los altos costos logís- } \\
\text { ticos en exportación e importación } \\
\text { de mercancías por contenedor uni- } \\
\text { tario. }\end{array}$} & Está conectado con más de 600 puertos en 140 países y principales líneas navieras a nivel mundial. \\
\hline & $\begin{array}{l}\text { La cercanía al Canal del Dique le permite movilizar cargas con volúmenes elevados que reducen los costos } \\
\text { logísticos en un } 30 \% \text { y } 38 \% \text { en comparación con el transporte terrestre y asistencia satelital. }\end{array}$ \\
\hline $\begin{array}{l}\text { Desarticulación en cuencas territo- } \\
\text { riales deficientemente comunica- } \\
\text { das. }\end{array}$ & $\begin{array}{l}\text { La cinco mayores empresas del sector, cuenta con un sistema virtual para el seguimiento de las actividades } \\
\text { en el terminal marítimo y terrestre, el cual permite por medio virtual (página web) que el cliente supervise el } \\
\text { estado de su carga }\end{array}$ \\
\hline $\begin{array}{l}\text { Especialización en mayor medida } \\
\text { en carga de contenedores. }\end{array}$ & $\begin{array}{l}\text { El puerto cuenta con } 8 \text { muelles, equipos tecnológicos, como grúas, plataformas, montacargas, etc., que le } \\
\text { permiten movilizar los contenedores de manera adecuada y eficiente. }\end{array}$ \\
\hline $\begin{array}{l}\text { Pocas relaciones comerciales y } \\
\text { estratégicas con los sectores lo- } \\
\text { gísticos de las ciudades portuarias } \\
\text { del país. }\end{array}$ & $\begin{array}{l}\text { El puerto posee seis grúas tipo pórtico y en los próximos años obtendrá una adicional, lo que permitirá un } \\
\text { incremento en la capacidad de su plataforma. }\end{array}$ \\
\hline
\end{tabular}

\begin{tabular}{|c|c|}
\hline Amenaza & Oportunidades \\
\hline $\begin{array}{l}\text { Problemas con los sedimentos de } \\
\text { tierra que arrastra el río Magda- } \\
\text { lena por el Canal del Dique, lo } \\
\text { cual resta profundidad al puerto y } \\
\text { poniendo en riesgo la efectividad } \\
\text { del Canal de Varadero. }\end{array}$ & $\begin{array}{l}\text { Firma de tratados de cooperación con otros puertos, los cuales permitan compartir experiencias, generar } \\
\text { alianzas de cooperación. }\end{array}$ \\
\hline $\begin{array}{l}\text { Dependencia del feedering por } \\
\text { proximidad a Panamá }\end{array}$ & $\begin{array}{l}\text { Oportunidades de negocios para clientes, generando un ambiente seguro para la carga de las líneas navieras } \\
\text { para ser competitivo en el mercado portuario, viendo en el puerto una oportunidad de crecimiento e inver- } \\
\text { sión. }\end{array}$ \\
\hline $\begin{array}{l}\text { Concesión de puertos ecuatorianos } \\
\text { (Manta, Guayaquil, Esmeraldas) a } \\
\text { operadores multinacionales }\end{array}$ & $\begin{array}{l}\text { Trabajar de la mano con el apoyo del Gobierno, para generar sinergia y crear mejores oportunidades de } \\
\text { negocios con otros países. }\end{array}$ \\
\hline $\begin{array}{l}\text { Posible apertura de otro canal } \\
\text { centro americano que comunique } \\
\text { al Océano Pacífico y Atlántico. }\end{array}$ & $\begin{array}{l}\text { Es uno de los puertos que más atención a recibido por parte de los inversionistas, por su imagen internacio- } \\
\text { nal, es visto como el portal de entrada al país, poniéndolo a la vanguardia de los puertos más avanzados en } \\
\text { materia tecnológica y de control. }\end{array}$ \\
\hline $\begin{array}{l}\text { Posicionamiento de China y otros } \\
\text { países emergentes en USA a través } \\
\text { de plataformas intermodales en } \\
\text { México }\end{array}$ & $\begin{array}{l}\text { La transformación tecnológica que ha experimentado el puerto en los últimos años, puede consolidarlo como } \\
\text { el Centro Logístico Integrado y de Trasbordo Internacional para el Caribe, y uno de los } 30 \text { mejores mega } \\
\text { puertos del mundo en } 2020 \text {. }\end{array}$ \\
\hline \multirow{3}{*}{$\begin{array}{l}\text { Múltiples iniciativas de desarrollo } \\
\text { portuario en la costa pacífica del } \\
\text { continente, con la adaptación de } \\
\text { infraestructura para la recepción de } \\
\text { grandes buques. }\end{array}$} & $\begin{array}{l}\text { Con la inversión realizada ( } 1.000 \text { millones de dólares), podrá duplicar los contenedores que actualmente } \\
\text { moviliza. }\end{array}$ \\
\hline & $\begin{array}{l}\text { Con la ampliación del Canal de Panamá y la inversión en infraestructura que se están realizando, podrá } \\
\text { recibir buques Post Panamax, los cuales tienen la capacidad de trasladar hasta } 14.000 \text { contenedores. }\end{array}$ \\
\hline & $\begin{array}{l}\text { En el puerto, se proyecta la implementación de degrado a mayor profundidad, con el fin de poder recibir } \\
\text { barcos de } 14 \text { a } 16 \text { metros de calado. }\end{array}$ \\
\hline
\end{tabular}

Fuente: elaboración propia con base en Organización Puerto de Cartagena; revisión sistemática de la literatura.

Por lo anterior, es importante también prestar especial atención a la preservación e integración del Canal del Dique, para mantener buenas actividades dentro de las plataformas y no lesionar la cadena en alguno de sus aspectos. Reducir los costos de transporte, dar mayor valor a agregado a los servicios que presta el puerto y tener vías alternativas como lo es este canal, son unas de las ventajas competitivas que se pueden apreciar aquí. 
En general, se pude decir que el puerto de Cartagena entra a desempeñar un papel importante, trascendental en el comportamiento económico de la región y del país en los próximos años, siempre y cuando se adopten las estrategias necesarias y pertinentes para poder cumplir con este compromiso y configurarse como Centro de Conexión Global por excelencia en el Caribe.

\section{Conclusiones}

Los principales hallazgos e implicaciones durante esta investigación, teniendo en cuenta la construcción de una matriz DOFA:

- Aún persisten problemas de movilidad en el corredor de carga con la mayoría de las empresas del sector logístico portuario a pesar de las inversiones en los últimos tres años (2013-2015) en materia de infraestructura y tecnología, para corregir esta situación y aumentar la competitividad.

- Las empresas de transporte de carga interna, deben trabajar mucho en la reducción en los costos de fletes dado que, comparado a ciudades como Barranquilla y Santa Marta, las empresas no están siendo competitivas a pesar de que Cartagena sea líder en el cargue y descargue de contenedores. Sin embargo, empresas como CONTECAR, Muelles del Bosque y Puerto Mamonal, han trabajado en ese aspecto y han logrado reducir sus costos.

- El aprovechamiento de la cercanía con el Canal del Dique y el Rio Magdalena por parte de las empresas que conforman el sector logístico portuario de Cartagena, debe potencializarse e incrementarse en la navegación y transporte de mercancía.

- La participación de las empresas del sector logístico portuario de la ciudad de Cartagena en los mercados nacionales e internacionales se ha visto amenazada. A nivel nacional, las concesiones por parte de la ANI en los último cinco años (20112015). Y a nivel internacional, la concesión de puertos ecuatorianos, el posicionamiento de China, USA y la dependencia del feedering en Panamá interfieren en mayor participación del mercado. De esto modo, las empresas de Cartagena se han protegido de esto gracias a los tratados de cooperación con otros puertos y la búsqueda de mayor apoyo por parte de la Agencia Nacional de Infraestructura (ANI).

- La ventaja de las empresas del sector logístico portuario de Cartagena sobre los demás sectores en el Caribe y a nivel nacional, radica en que la conectividad con más de 600 puertos. Esto es como resultado de los estándares de calidad que maneja el sector de Cartagena, y que impide y complica la instalación de otras empresas por la alta exigencia en la dimensión de competitividad. De este modo, se refleja como Cartagena ha aprovechado sus ventajas competitivas y al mismo tiempo deja ver la falta de aprovechamientos por otros sitios en el caribe.

\section{Recomendaciones}

Teniendo en cuenta los resultados del presente estudio, surgen algunas recomendaciones de políticas a las empresas que conforman el sector logístico portuario de la ciudad, tales como:

- Las empresas del sector deben implementar políticas y planes que busquen la articulación del puerto con otros nodos de transporte (multimodal) a nivel nacional, ya que se presenta un desajuste estructural; estando uno de los principales puertos en Cartagena (en la costa) y los principales centros de producción y consumo ubicados en el interior del país.

- Por otra parte, una recomendación pertinente a los agentes de este sector es que, canalicen recursos a tecnologías virtuales (plataformas de servicios, cotizaciones, acompañamiento y verificación del estado de mercancías), ya que con el boom tecnológico y la nueva era digital, el interés de las empresas internacionales por hacer uso de nuevas tecnologías portuarias será mayor, lo que representa una debilidad sino cuentan con estos servicios, pero puede ser una gran fortaleza si le apuntan a este rubro.

- Según las proyecciones del Banco Mundial, a 2020, los costos del transporte de Anfibios ISSN: 2665-1513 
mercancías disminuirán en un 50\%, por tanto, una recomendación importante es que, identifiquen los rubros que generan mayor costo y apunten al mejoramiento de estos mismos, para que en el mediano plazo los costos de los servicios ofrecidos no sean mayores a los ofrecidos por los competidores y así puedan mantener la ventaja competitiva.

- Otra recomendación en materia de políticas es la construcción y adecuación pronta del Canal El Varadero, principalmente por dos razones; la primera es que, con la construcción de este canal se puede ampliar el Canal del Dique para poder responder al incremento de la demanda del puerto por la ampliación del Canal de Panamá; y la segunda porque no cuenta con una vía alterna que descongestione dicho tráfico, e incluso, en ocasiones afecta otras vías de la ciudad, principalmente las del norte.

- Otra recomendación es que, apunten a las relaciones portuarias con los puertos y empresas brasileñas, ya que el comportamiento económico de Brasil es cada día mayor, adicionalmente es uno de los países con mayor consumo en Sur América, es decir esta región es potencialmente consumidora de productos colombianos, principalmente los que tiene que ver con la agroindustria, manufactura, sector textil y servicios. En otras palabras, Brasil representa un mercado potencial relevante para Colombia, ya que cuenta con más de 40 millones de habitantes de clase media, con un fuerte rezago en infraestructura portuaria, permitiéndole a Colombia, a través del puerto de Cartagena, atender de manera eficiente los cinco puertos de Brasil.

\section{Referencias bibliográficas}

Acosta, K. (2012). Cartagena entre el progreso industrial y el rezago social, (Núm. 178), 64. Retrieved from http://www.banrep.gov.co/docum/Lectura_finanzas/pdf/dtser_178.pdf

Amaya, J. (2010). El método DOFA, un método muy utilizado para diagnóstico de vulnerabilidad y planeación estratégica. El Prisma, 1-7. Ediciones EFIM
Retrieved from http://www.elprisma.com/apuntes/administracion_de_empresas/metododofanociones/default2.asp

ANDI. (2015). Boletín N²-202. Gerencia de Logística, Transporte e Infraestructura. Información del Sector. Bogotá, Colombia.

Andrews, K. (1971). The Concept of Corporate Strategy. Innovar, 19(35), 167-169. Retrieved from http://www.scielo.org.co/scielo.php?scrip$\mathrm{t}=$ sci_arttext\&pid=S0121-50512009000300012 Bejarano Morales, F. (2013). Estudios de Mercado. Sector Portuario Colombiano e Incidencia de las Políticas Públicas en la SPRBUN. Delegatura de Protección de la Competencia. Bogotá, Colombia.

Martínez Ortiz, A., Malagón, J., \& Muñoz, I. (2014). Impacto económico y social del puerto de Cartagena. Bogotá, Colombia. Retrieved from http://www.fedesarrollo.org.co/wp-content/uploads/Informe-Final-SPRC_final.pdf

Mintzberg, H. (1985). Of strategies, deliberate and emergent. Strategic Management Journal, 6(3), 257-272. https://doi.org/10.1002/ smj.4250060306

PNUD, P. de la N. U. para el D. (2014). Perfiles ocupacionales del sector logístico/portuario de la ciudad de Cartagena de Indias-2013. Bogotá, Colombia.

Ponce, H. (2007). La Matriz Foda : Alternativa De Diagnóstico Y Determinación De Estrategias De Intervención En Diversas Organizaciones. Enseñanza E Investigación En Psicología, 12(55), 113-130.

Ramírez-Rojas, J. L. (2009). Procedimiento para la elaboración de un análisis FODA como una herramienta de planeación estratégica en las empresas. Ciencia Administrativa, 2, 54-61. Rivera, H., \& Malaver, M. (2011). ¿Qué estudia la estrategia? Documentos de Investigación, 99(99), 1-22.

Rodriguez, C. (2013). Análisis del transporte de carga en Colombia, para crear estrategias que permitan alcanzar estándares de competi- 
Diógenes Alejandro Arrieta Rodríguez, Diego A. Cardona A.

tividad e infraestructura internacional. Tesis de Grado, Universidad Del Rosario, 77. Retrieved from http://medcontent.metapress.com/index/ A65RM03P4874243N.pdf

Selznick, P. (1957). Leadership in administration : a sociological interpretation. University of California Press.

World Bank Group. (2016). Doing Business 2016. Measuring Regulatory Quality and Efficiency. Washington D.C. https://doi. org/10.1596/978-1-4648-0667-4

World Economic Forum, W. (2015). The global competitiveness report 2015-2016. World Economic Forum (Vol. 5). Geneva. https://doi. org/92-95044-35-5 